\title{
Skin Necrosis After Cytoreductive Surgery And Hyperthermic İntraperitoneal Chemotherapy: A Case Report
}

\section{Sitoreduktif Cerrahi Ve Hipertermik İntraperitoneal Kemoterapi Sonrası Nadir Görülen Bir Komplikasyon; Cilt Nekrozu, Olgu Sunumu}

\author{
Eda Güner, Serdar Culcu, Ferit Aydin \\ SBU Dr Abdurrahman Yurtaslan Ankara Onkoloji Eğitim ve Araştırma Hastanesi, Genel Cerrahi Kliniği
}

Dergiye Ulaşma Tarihi: Dergiye Kabul Tarihi: Doi: 10.5505/aot.2020.41275

\section{ÖZET}

Peritoneal karsinomatozisi olan seçilmiş hasta grubunda sitoreduktif cerrahi ve hipertermik intraperitoneal kemoterapi oldukça etkili bir tedavi metodudur. Bu strateji pseudomiksoma peritonei, peritoneal mezotelyoma gibi hastalıklarda uzun dönem sonuçları oldukça iyidir. Overin epitelyal tümörlerine, koleraktal kanserlerin peritoneal metastazlarında da faydalı olduğunu gösteren çalışmalar mevcuttur. Bir çok iyi sonucunun yanında sitoreduktif cerrahi ve hipertermik intraperitoneal kemoterapinin mortalitesi ve morbiditesi oldukça yüksektir. Bu makalemizde sitoreduktif cerrahi ve hipertermik kemoterapi sonrası gelişen oldukça nadir görülen cilt nekrozu komplikasyonunu sunduk.

Anahtar Kelimeler: sitoreduktif cerrahi, hipertermik intraperitoneal kemoterapi, peritoneal karsinomatozis

\begin{abstract}
Cytoreductive surgery and hyperthermic intraperitoneal chemotherapy is a very effective treatment combination in selected patients with peritoneal carcinomatosis. This protocol has good long-term results in diseases such as pseudomyxoma peritonei and peritoneal mesothelioma. There are also studies that show the benefits of cytoreductive surgery and hyperthermic intraperitoneal chemotherapy in ovarian epithelial tumors and peritoneal metastasis of colorectal cancers. However mortality and morbidity rates of cytoreductive surgery and hyperthermic intraperitoneal chemotherapy are high. In this report, we presented a very rare case, skin necrosis that occured after cytoreductive surgery and hyperthermic intraperitoneal chemotherapy.
\end{abstract}

Keywords: Cytoreductive surgery, hyperthermic intraperitoneal chemotherapy, peritoneal carcinomatosis

\section{Gİiș}

Özelleşmiş merkezlerde primer ve sekonder peritoneal karsinomatozda seçilmiş hasta grubunda sitoreduktif cerrahi (SRC) ve hipertermik intraperitoneal kemoterapi (hipek) kombinasyonuna bağlı oldukça başarılı sonuçlar bildirilmiştir. Teorik olarak peritoneal karsinomatozda SRC makroskopik hastalığ HIPEK ise mikroskobik hastalı̆̆ı tedavi eder. Bu kombinasyon pseudomiksoma peritonei ve peritoneal mezotelyomada standart tedavi protokolunu oluşturur (1-4). Ayrica koleraktal kanserlere bağlı gelişen ve overin seroz kistadenokarsinomuna bağl1 gelişen peritoneal karsinomatozda da bu tedavi yaklaşımının etkinliğini bildiren çalışmalar mevcuttur (5-8). Yapılan cerrahinin genişliği, kullanılan sitotoksik ajanlar, hastalığın yaygınlı $\breve{g}$, daha önce geçirilmiş cerrahiler, kansere bağlı malnutrisyon gibi nedenlerle SRC ve HIPEK beraberinde artmıs mortatlite ve morbiditeyi getirir (9). Ayrıca bu ileri evre hastalığa sahip hastaların bir çoğu daha önceden kemoterapi almış olup metabolik, immun, endokrinolojik disfonksiyonel nutrisyonel bozukluklara sahip olması da mortaliteyi etkileyen faktörler arasındadır (9). Bu girişimler sırasında veya sonrasinda gastrointestinal, pulmoner, 
hemotalojik, enfeksiyoz komplikasyonlar gibi birçok komplikasyon görülebilir.

$\mathrm{Bu}$ yazıda; overin seröz karsinomuna bağlı peritoneal karsinomatozisde, sitoreduktif cerrahi ve hipertermik intraperitoneal kemoterapi sonrası nadir görülen cilt nekrozu gelişen hastamızı güncel literatürler eşliğinde sunduk.

\section{OLGU SUNUMU}

50 yaşında kadın hasta karın ağrısı karında şişlik şikayetleri ile dış merkezde asit nedeniyle tetkik edilen hastanın sitoloji sonucu serözkarsinom ile uyumlu malign bulgular gelmesi üzerine kliniğimize kliniğimize interne edildikten sonra preoperatif hazırlıkları yapıldı. Özgeçmişinde 2 yıl önce apendektomi mevcut bunun dışında bilinen bir hastalığı yok. Ameliyat öncesi yapılan görüntülemelerinde Peritoneal karsinomatoz index (PCI) skoru 9. Ameliyat öncesi alınan tümör belirteçleri CA$125=191.6, \quad \mathrm{CEA}=0,14, \quad \mathrm{CA} 15-3=35,9$. Hastanın yapılan operasyonunda çıkan kolon transvers kolon inen kolon serozasinda ve transvers kolon mezosunda milimetrik tümöral ekilimler batın yan duvarında milimetrik ekilimler omentum üzerinde tümöral ekilimler mevcuttu. Hastaya total abdominal histerektomi ,ooferektomi,total peritonektomi, omentektomi, subtotal kolektomi ileorektal anostomoz, koruyucu ileostomi açıldı. Hastaya preoperatif 42 santigrad derecede 1 saat boyunca $20 \mathrm{mg}$ mitomycin, carboplatin $450 \mathrm{mg}$ ile perfuze edildi. Ameliyat süresi 255 dakika idi. Ameliyat esnasında hastaya 1 ünite eritrosit süspansiyonu 2 ünite taze donmuş plazma verildi. Postoperatif 1. günde hastaya enteral beslenme başlandi. Postoperatif 4. günde hastanın yara yerinde ani gelişen yaklaşık 5-6 cm çapında cilt nekrozu gelişti (şekil 1-2). Hasta debritman yapıldıktan sonra etkilenen bölgeden ve debrite edilen cilt kısmından yara kültürü alındı. Herhangi bir üreme saptanmadı. Yara sekonder iyileşmeye bırakıld1 ve hasta postoperatif 14. Gününde problemsiz taburcu edildi. Hasta halen takibimizde olup sorunsuz olarak adjuvan kemoterapi almaktadır.

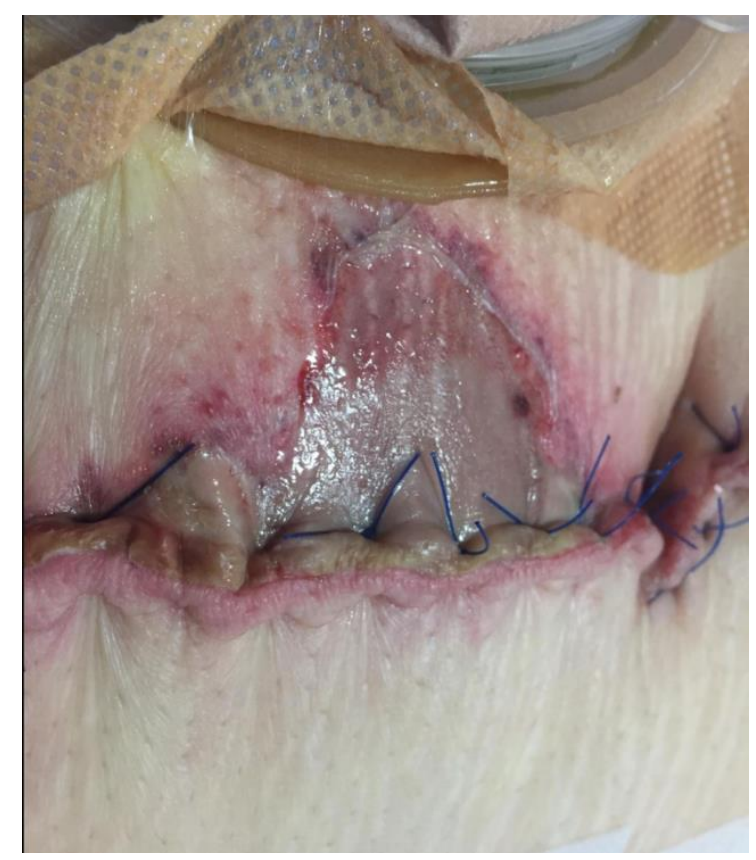

Şekil 1. Yara yerinde görülen cilt nekrozu

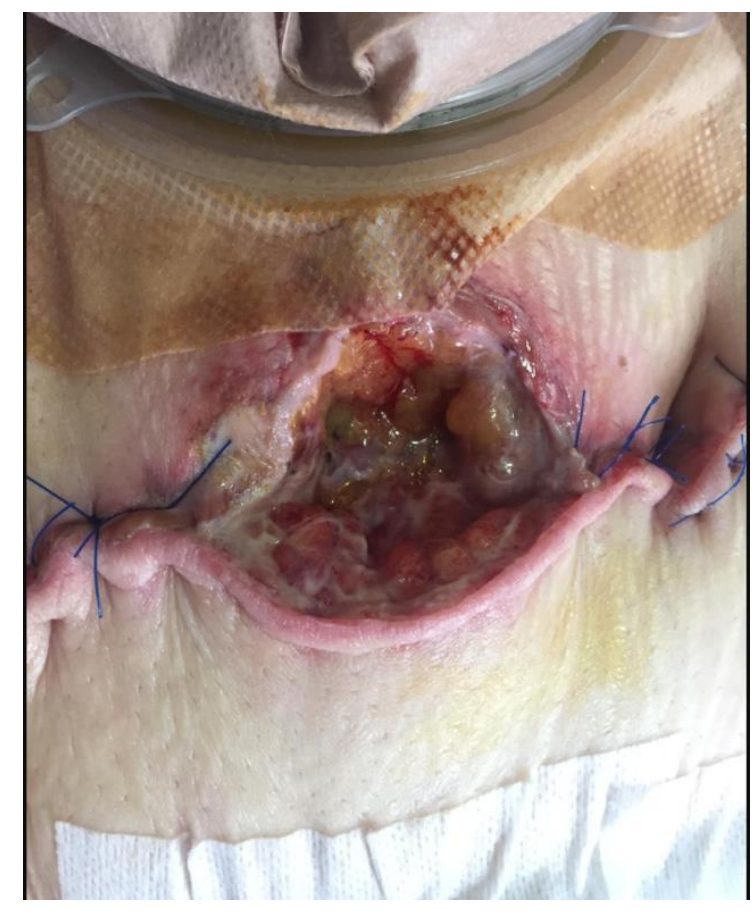

Şekil 2. Cilt nekrozu debritmanı sonrası

\section{TARTIŞMA}

Özelleşmiş merkezlerde primer ve sekonder peritoneal karsinomatozda seçilmiliş hasta grubunda sitoreduktif cerrahi (SRC) ve hipertermik intraperitoneal kemoterapi (hipek) kombinasyonuna bağlı oldukça başarılı sonuçlar bildirilmiştir. Teorik olarak peritoneal karsinomatozda SRC makroskopik hastalı̆ 1 , HIPEK mikroskobik hastalığı tedavi eder. Bu 
kombinasyon pseudomiksoma peritonei ve peritoneal mezotelyoma da standart tedavi protokolunu oluşturur (1-4). Ayrıca koleraktal kanserlere bağlı gelişen, overin epitelyal tümörlerine bağlı gelişen peritoneal karsinomatozda da bu tedavi yaklaşımının etkinliğini bildiren çalışmalar mevcuttur (5-8).

Yapılan cerrahinin genişliği, kullanılan sitotoksik ajanlar, hastalığın yaygınlığı, daha önce geçirilmiş cerrahiler, kansere bağlı malnutrisyon gibi nedenlerle SRC ve HIPEK beraberinde artmış mortatlite ve morbiditeyi getirir (9). Ayrica bu ileri evre hastalığa sahip hastaların bir çoğu daha önceden kemoterapi almış olup metabolik, immun, endokrinolojik disfonksiyonel nutrisyonel bozukluklara sahip olmas1 da mortaliteyi etkileyen faktörler arasindadir (9).

Epitelyal over kanseri kadınlarda görülen kanserlerin küçük bir kısmını oluşturur, tüm kanserler arasında 17. sırada görülür (10). Epitelyal over kanserleri postmenopozal dönemde genelde yaşamın 6-7. dekatında görülür. Epitelyal over kanserinde \%60-70 tanı anında peritoneal karsinomatozis mevcuttur (11).

Epitelyal over kanserinde ana prognostik faktörler hastanın yaşına, hastalığın yaygınlığ 1 , kemoterapiye yanıt, tümör evresi ve sitoreduktif cerrahi sonrası rezidüel hastalıktır (12). Armstrong ve arkadaşlarının ileri evre epitelyal over tümörlü hastalarda cerrahi ile kombine edilen 1v kemoterapi ve cerrahi ile kombine edilen hipertermik intraperitoneal kemoterapiyi karşılaştırdıkları çalışmada 1. Grupta ortalama sağkalımı 49 ay 2. Grupta ise 65.6 ay bulmuşlardır (13). Bir çok çalışmada hiperterminin tümör hücrelerinde apopitozisi arttırdığı, kemoterapotik ilaçların etkinliğini arttırdığı gösterilmiştir (14).

Epitelyalover kanserinde SRC ve HIPEK sonras1 mortalite \%2-10'dur (15). Operasyon sonrası özellikle pulmoner komplikasyonlar, barsak perforasyonu ve nekrozu, yara yeri enfeksiyonları, nefrototoksisite açısından dikkatli olunmalıdır ve morbidite oranlar1 \%10-40'tır (16).

Epitelyal over kanserleri için HIPEK uygulama süresi standart olmayıp tercihen 60120 dakikadır. Giriş 1sıs1 39-43.6 santigradderece, çıkış 1sısı 36.9-42.9 santigrad derece uygulanabilir (17). İlaç protokolü olarak cisplatin $50-100 \mathrm{mg} / \mathrm{m}^{2}$, carboplatin $350-1000$ $\mathrm{mg} / \mathrm{m}^{2}$, paclitaxel $60-175 \mathrm{mg} / \mathrm{m}^{2}$, ya da docetaxel $75 \mathrm{mg} / \mathrm{m}^{2}$ tek ajan olarak verilebilir. Cisplatindoce-aksel ya da mitomycin $\mathrm{C}$ ile kombine eden uygulayıcılarda vardır (18). Biz klinik olarak mitomycinC ,carboplatin kombinasyonunu 42 derece 1 saat perfuzyon olarak tercih ediyoruz.

\section{SONUÇ}

Sitoreduktif cerrahi ile birlikte uygulanan hipertermik intraperitoneal kemoterapinin başarılı sonuçları bildirilmiş olsa da mortalitesi ve morbiditesi oldukça yüksek bir girişimdir. Komplikasyonlara karşı cerrahi ekip hazırlıklı olmalı gelişebilecek komplikasyonları öngörebilmeli ve önlemini almalıdır. Bu makalemizde SRC ve HIPEK sonrası gelişen cilt nekrozu ve tedavi yaklaşımımızı sunduk.

\section{REFERANSLAR}

1. Sugarbaker PH (2006) New standard of care for appendiceal epi- thelial neoplasms and pseudomyxoma peritonei syndrome? Lancet Oncol 7(1):69-76

2. Chua TC, Moran BJ, Sugarbaker PH, Levine EA, Glehen O, Gilly FN, et al. (2012) Early- and longterm outcome data of patients with pseudomyxoma peritonei from appendiceal origin treated by a strategy of cytoreductive surgery and hyperthermic intraperitoneal che- motherapy. J Clin Oncol 30(20):2449-2456

3. Yan T, Welch L, Black D, Sugarbaker P (2007) A systematic review on the efficacy of cytoreductive surgery combined with periopera- tive intraperitoneal chemotherapy for diffuse malignancy peritoneal mesothelioma. Ann Oncol 18(5):827-834

4. Deraco M, Nonaka D, Baratti D, Casali P, Rosai J, Younan R, et al. (2006) Prognostic analysis of clinicopathologic factors in 49 pa- tients with diffuse malignant peritoneal mesothelioma treated with cytoreductive surgery and intraperitoneal hyperthermic perfusion. Ann Surg Oncol 13(2):229237

5. Verwaal V, Bruin S, Boot H, van Slooten G, van Tinteren H (2008) 8-year follow-up of randomized trial: cytoreduction and hyperther- mic intraperitoneal chemotherapy versus systemic chemotherapy in patients with peritoneal carcinomatosis of colorectal cancer. Ann Surg Oncol 15(9):2426-2432

6. Glehen O, Schreiber V, Cotte E, Sayag-Beaujard AC, Osinsky D, Freyer G, et al. (2004) Cytoreductive surgery and intraperitoneal chemohyperthermia for peritoneal carcinomatosis arising from gas- tric cancer. Arch Surg 139(1):20-26

7. Elias D, Lefevre JH, Chevalier J, Brouquet A, Marchal F, Classe JM, et al. (2009) Complete cytoreductive surgery plus intraperito- neal chemohyperthermia with oxaliplatin for peritoneal carcinoma- tosis of colorectal origin. J Clin Oncol 27(5):681-685 
8. Shen P, Thai K, Stewart JH, Howerton R, Loggie BW, Russell GB, et al. (2008) Peritoneal surface disease from colorectal cancer: com- parison with the hepatic metastases surgical paradigm in optimally resected patients. Ann Surg Oncol 15(12):3422-3432

9. Stephens AD, Alderman R, Chang D, et al.: Morbidity and mortality of 200 treatments with cytoreductive surgery and hyperthermic intraoperative intraperitoneal chemotherapy using the Coliseum technique. Ann Surg Oncol 1999;6:790796.
10. https://www.cancer.gov/about-cancer/understanding/statistics

11. Peiretti M, Zanagnolo V, Aletti GD, Bocciolone L, Colombo N, Landoni $F$, et al. Role of maximal primary cytoreductive surgery in patients with advanced epithelial ovarian and tubal cancer: Surgical and oncological outcomes. Single institution experience. Gynecol Oncol 2010;119(2):259-64

12. Narasimhulu DM, Khoury-Collado F, Chi DS. Radical surgery in ovarian cancer. Curr Oncol Rep 2015;17(4):16. 